\title{
Comparison of the toxicities, bioactivities and chemical profiles of raw and processed Xanthii Fructus
}

\author{
Tao Su', Brian Chi-Yan Cheng ${ }^{1}$, Xiu-Qiong Fu', Ting Li ${ }^{1}$, Hui Guo ${ }^{1}$, Hui-Hui Cao ${ }^{1}$, Hiu-Yee Kwan', \\ Anfernee Kai-Wing Tse ${ }^{1}$, Hua Yu' ${ }^{1}$, Hui $\mathrm{CaO}^{2}$ and Zhi-Ling $\mathrm{Yu}^{1,3^{*}}$
}

\begin{abstract}
Background: Although toxic, the Chinese medicinal herb Xanthii Fructus (XF) is commonly used to treat traditional Chinese medicine (TCM) symptoms that resemble cold, sinusitis and arthritis. According to TCM theory, stir-baking (a processing method) can reduce the toxicity and enhance the efficacy of XF.

Methods: Cytotoxicities of raw XF and processed XF (stir-baked XF, SBXF) were determined by the MTT (3-(4,5-dimethylthiazol-2-yl)-2,5-diphenyltetrazolium bromide) assay in normal liver derived MIHA cells. Nitric oxide (NO) production and inducible nitric oxide synthase (iNOS) mRNA expression were measured by the Griess reagent and quantitative real-time PCR, respectively. The chemical profiles of XF and SBXF were compared using an established ultra-performance liquid chromatography/quadrupole-time-of-flight mass spectrometry (UPLC/Q-TOF-MS) method.

Results: SBXF was less toxic than XF in MIHA cells. Both XF and SBXF could reduce NO production and iNOS mRNA expression in lipopolysaccharide-stimulated RAW 264.7 macrophages. Interestingly, the effects of SBXF were more potent than XF in the macrophages. By comparing the chemical profiles, we found that seven peaks were lower, while nine other peaks were higher in SBXF than in XF. Eleven compounds including carboxyatractyloside, atractyloside and chlorogenic acid corresponding to eleven individual changed peaks were tentatively identified by matching with empirical molecular formulae and mass fragments, as well as literature data.

Conclusion: Our study showed that stir-baking significantly reduced the cytotoxicity and enhanced the bioactivity of XF; moreover, with a developed UPLC/Q-TOF-MS method we differentiated XF and SBXF by their chemical profiles. Further studies are warranted to establish the relationship between the alteration of chemical profiles and the changes of medicinal properties caused by stir-baking.
\end{abstract}

Keywords: Xanthii Fructus, Stir-baking, Cytotoxicity, Anti-inflammation, UPLC/Q-TOF-MS

\section{Background}

Xanthii Fructus (XF) is the ripe fruits of Xanthium sibiricum Patr. (Compositae). In Sheng Nong's herbal classic (a book published 2,000 years ago), XF was first documented to be able to smooth nasal orifices (通鼻䛎) and eliminate wind-dampness (祛风湿), and to be toxic. It is commonly used in managing traditional Chinese medicine (TCM) symptoms that would today be diagnosed as cold,

\footnotetext{
* Correspondence: zlyu@hkbu.edu.hk

'School of Chinese Medicine, Hong Kong Baptist University, Kowloon Tong, Hong Kong, China

${ }^{3}$ Institute of Integrated Bioinfomedicine \& Translational Science, HKBU Shenzhen Research Institute and Continuing Education, Shenzhen, China

Full list of author information is available at the end of the article
}

sinusitis and arthritis [1]. To reduce the toxicity and enhance the efficacy, XF is usually processed by stir-baking (炒). In the Chinese pharmacopoeia, more than ten Chinese proprietary drugs contain XF or stir-baked XF (SBXF). Chemical analyses revealed that XF contains water-soluble glycosides, sesquiterpene lactones and phenolic acids [2]. Pharmacological studies showed that XF has various bioactivities including anti-oxidant [3], anti-bacterial [4] and anti-inflammatory [5] properties. In a carrageenan-induced hind paw edema rat model, $\mathrm{XF}$ was shown to have anti-inflammatory effect as demonstrated by reducing the levels of inducible nitric oxide synthase (iNOS) and cyclooxygenase-2 (COX-2) 
expressions [5]. In an acetic acid-induced writhing model, SBXF was shown to have better analgesic effect than XF in mice [6]. Toxicological studies demonstrated that XF induced obvious liver damage in a longterm toxicity study in rats [7], the water extract was more toxic than the ethanol extract of XF in mice $[8$, 9], and the water extract of SBXF was less toxic than $\mathrm{XF}$ in an acute toxicity study in mice [6]. However, the comparison of the hepatotoxicities of XF and SBXF has not been conducted. Proteins and water-soluble glycosides have been thought to be the main toxic substances of this herb. Denaturing the toxic proteins by stir-baking has been proposed as one of the reasons for reducing its toxicity [10]. The water-soluble glycosides carboxyatractyloside (CATR) and atractyloside (ATR) have been recognized as other two toxic components of this herb $[11,12]$.

To validate the impact of stir-baking on the toxicity and efficacy, in this study, we compared the cytotoxicities of XF and SBXF in non-tumorigenic and immortalized human liver cells (MIHA), and their effects on the production of an inflammatory mediator nitric oxide (NO) in lipopolysaccharide (LPS)-stimulated Raw 264.7 macrophages. In an attempt to uncover the chemical basis behind the potential changes of medicinal properties caused by stir-baking, we compared the chemical profiles of the water extracts of XF and SBXF using an established UPLC/Q-TOF-MS method.

\section{Methods}

\section{Chemicals and regents}

Carboxyatractyloside potassium salt $\left(\mathrm{C}_{31} \mathrm{H}_{43} \mathrm{O}_{18} \mathrm{~S}_{2} \mathrm{~K}_{3}\right)$ was purchased from Merck (Merck Millipore, Taiwan). Atractyloside potassium salt $\left(\mathrm{C}_{30} \mathrm{H}_{44} \mathrm{O}_{16} \mathrm{~S}_{2} \mathrm{~K}_{2}\right)$, chlorogenic acid $\left(\mathrm{C}_{16} \mathrm{H}_{18} \mathrm{O}_{9}\right)$, caffeic acid $\left(\mathrm{C}_{9} \mathrm{H}_{8} \mathrm{O}_{4}\right)$, 1,5-O-Dicaffeoylquinic acid $\left(\mathrm{C}_{25} \mathrm{H}_{24} \mathrm{O}_{12}\right)$, 3-(4,5-dimethylthiazol-2-yl)-2,5-diphenyltetrazolium bromide (MTT), bacterial lipopolysaccharide (LPS) and Griess reagent were purchased from Sigma-Aldrich (Sigma, USA). All solvents for chemical analyses were purchased from RCI Labscan Ltd. (Thailand). All materials for cell culture were obtained from Life Technologies Inc. (GIBICO, USA).

\section{Preparation of XF and SBXF extracts}

Ten batches of XF were collected from different places in China (01: Yulin, Shanxi province; 02: Shunyi, Beijing; 03: Houma, Shanxi province; 04-05: Baoding, Hebei province; 06: Handan, Hebei province; 07-10: Hong Kong), and their authentication were confirmed by the corresponding author. Voucher specimens were deposited at the School of Chinese Medicine, Hong Kong Baptist University.
SBXF preparation: Raw XF was stir-baked in a preheated wok. The processed herb is SBXF.

XF and SBXF extracts preparation: XF or SBXF $(100 \mathrm{~g})$ was reflux-extracted twice with water $(1: 10, \mathrm{w} / \mathrm{v})$ for $2 \mathrm{~h}$ each. The combined extracts were filtered and then concentrated under reduced pressure to remove the water. The powdered XF (yield: $12.00 \%$ ) and SBXF (yield: $17.22 \%$ ) extracts were obtained by lyophilizing of the concentrated samples with a Virtis Freeze Dryer (The Virtis Company, New York, USA).

XF and SBXF fractions preparation: XF or SBXF $(100 \mathrm{~g})$ was reflux-extracted twice with $80 \%$ ethanol $(1: 10, \mathrm{w} / \mathrm{v})$ for $2 \mathrm{~h}$ each. The combined extracts were filtered and evaporated under vacuum, then suspended in water and partitioned successively with petroleum (PE), ethyl acetate (EA) and n-butanol $(\mathrm{n}-\mathrm{Bu})$. Each fractions of XF and SBXF were evaporated in a Virtis Freeze Dryer to yield the residues of PE (8.56\%), EA (5.99\%), n-Bu (16.7 \%) and aqueous (68.75\%) for XF, and $\mathrm{PE}$ (10.18\%), EA (7.28 \%), n-Bu (17.00 \%) and aqueous (65.54\%) for SBXF, respectively.

\section{Cell culture}

The non-tumorigenic and immortalized human liver cells (MIHA) and the murine macrophage cells (Raw 264.7) were obtained from the American Type Culture Collection (ATCC, Manassa, VA, USA). All cells were cultured in dulbecco's modified eagle medium (DMEM) supplemented with $10 \%$ heat inactivated fetal bovine serum and $1 \%$ penicillin/streptomycin at $37{ }^{\circ} \mathrm{C}$ in humidified $5 \% \mathrm{CO}_{2}$ atmosphere.

\section{Cytotoxicity assay}

MIHA cells were seeded on a 96-well plate $(5000$ cells/well) and allowed to adhere overnight. The cells were treated with various concentrations of the water extracts and fractions of XF and SBXF as indicated for $48 \mathrm{~h}, 20 \mu \mathrm{l}$ of MTT solution $(5 \mathrm{mg} / \mathrm{ml})$ was added to each well and incubated for an additional $3 \mathrm{~h}$. The formazan crystal formed was dissolved with $100 \mu$ l of dimethylsulfoxide (DMSO), absorbance was detected at $570 \mathrm{~nm}$ by a microplate spectrophotometer (BD Biosciences, USA). Results were expressed as percentages of the respective controls [13].

\section{Nitric oxide (NO) production assay}

RAW 264.7 cells were seeded on a 24-well plate $(1 \times$ $10^{5}$ cells/ well) and allowed to adhere overnight. After pretreated with LPS $(1 \mu \mathrm{g} / \mathrm{ml})$ for $2 \mathrm{~h}$, the cells were treated with different subtoxic concentrations of XF or SBXF water extract $(100,200,300 \mu \mathrm{g} / \mathrm{ml}$, cell survival $>90 \%$ ) in the presence of LPS for another $24 \mathrm{~h}$. $\mathrm{NO}$ production was determined by assaying the accumulation of nitrite, the primary stable breakdown 
product of $\mathrm{NO}$ in the culture medium, with the Griess reagent [14]. The absorbance at $540 \mathrm{~nm}$ was measured using a microplate spectrophotometer (BD, Bioscience USA).

\section{Real-time polymerase chain reaction}

RAW 264.7 cells were seeded as described in Section "Nitric oxide (NO) production assay". After pretreated with LPS $(1 \mu \mathrm{g} / \mathrm{ml})$ for $2 \mathrm{~h}$, the cells were treated with different subtoxic concentrations of XF or SBXF water extract $(100,200,300 \mu \mathrm{g} / \mathrm{ml}$, cell survival $>90 \%)$ in the presence of LPS for another $18 \mathrm{~h}$. Total RNA was isolated using Trizol reagent (Invitrogen, USA) according to manufacturer's protocol [15]. Five micrograms of RNA was used for reverse transcription by oligo-dT using the SuperScript II Reverse Transcription Kit (Invitrogen, USA). The primers were designed as follows: iNOS (Sense 5'-AACGGAGAACGTTGGA TTTG-3' and anti-sense 5'-CAGCACAAGGGGTTT TCTTC-3'). To normalize the amounts of RNA in samples, a PCR reaction was also performed with primers of GAPDH (Sense 5'-AACTTTGGCATTGTGGAAGG-3' and anti-sense 5'-TGTGAGGGAGATGCTC AGTG-3'). Real-time PCR was performed using SYBR green reaction mixture in the ABI 7500 Fast Real-time PCR System (Applied Biosystems, USA).

\section{UPLC/Q-TOF-MS analysis}

Liquid chromatography was performed on an Agilent 1200 system coupled with an ACQUITY UPLC HSS T3 column $(2.1 \mu \mathrm{mm} \times 100 \mathrm{~mm}, 1.8 \mu \mathrm{m})$ maintained at $35{ }^{\circ} \mathrm{C}$. Elution was performed with a mobile phase of A (0.1\% formic acid in acetonitrile) and B (0.1\% formic acid in water). A gradient elution of $12 \% \mathrm{~A}$ at $0-2 \mathrm{~min}, 12-25 \% \mathrm{~A}$ at $2-5 \mathrm{~min}, 25-40 \% \mathrm{~A}$ at $5-7.5 \mathrm{~min}, 40-65 \% \mathrm{~A}$ at $7.5-10 \mathrm{~min}, 65-80 \% \mathrm{~A}$ at 10-13 $\mathrm{min}$ and $80-12 \% \mathrm{~A}$ at $13-17 \mathrm{~min}$ was employed. The flow rate was set at $0.35 \mathrm{ml} / \mathrm{min}$. The injection volume was $5 \mu \mathrm{l}$.

Mass spectrometric detection was carried out on an Agilent 6540 Q-TOF mass spectrometer (Hewlett Packard, Agilent, USA) with electrospray ionization (ESI) interface. The negative ion mode was used with the mass range set at $\mathrm{m} / \mathrm{z} \quad 100-1700$. The conditions of ESI source were as follows: gas temperature, $300^{\circ} \mathrm{C}$; drying gas $\left(\mathrm{N}_{2}\right)$ flow rate, $8 \mathrm{~L} / \mathrm{min}$; nebulizer, $45 \mathrm{psi}$; sheath gas temperature, $350{ }^{\circ} \mathrm{C}$; sheath gas flow, $10 \mathrm{~L} / \mathrm{min}$; capillary voltage, $4000 \mathrm{~V}$; fragmentor, $140 \mathrm{~V}$; skimmer voltage, $65 \mathrm{~V}$; OctopoleRFPeak, 750 V. Data were collected with the LC-MS-QTOF MassHunter Data Acquisition Software Ver. A.01.00 (Agilent Technologies) and analyzed with the Agilent MassHunter Qualitative Analysis Software B.06.00, respectively.
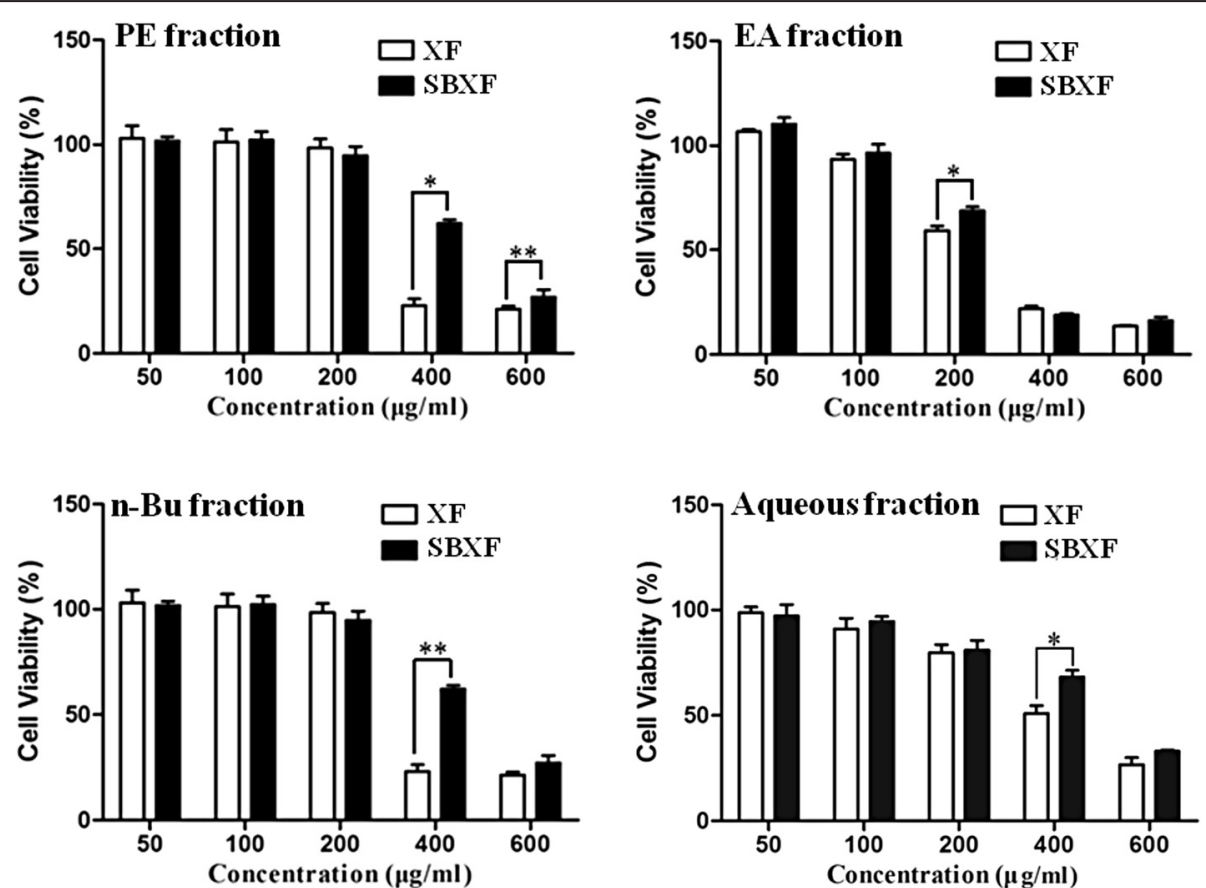

Fig. 1 Cytotoxicities of XF and SBXF fractions in cultured MIHA cells. MIHA cells were treated with various concentrations of XF and SBXF fractions as indicated for $48 \mathrm{~h}$, cell viability was determined by the MTT assay. All data were presented as mean \pm SD. PE: petroleum; EA: ethyl acetate; n-Bu: n-butanol. * $p<0.05,{ }^{* *} p<0.01$ 

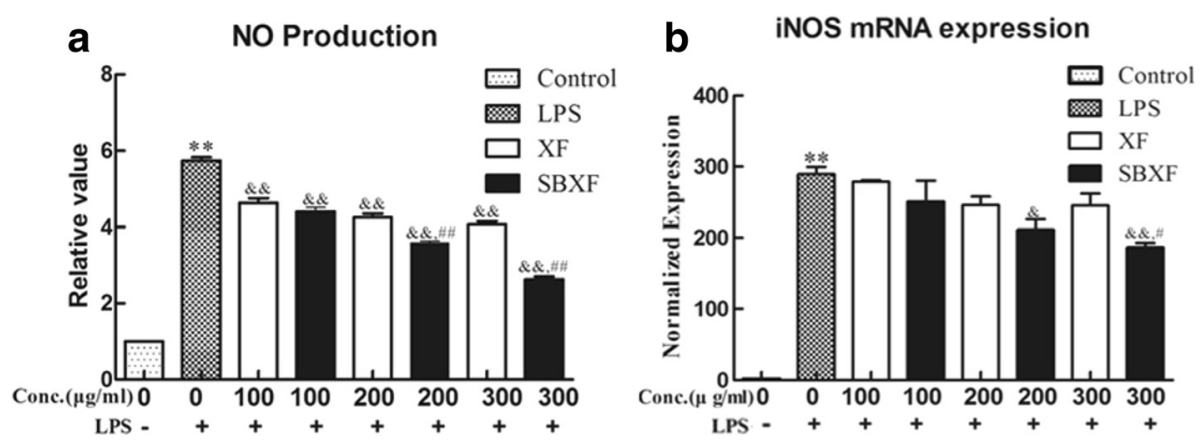

Fig. 2 Effects of XF and SBXF on NO production (a), iNOS mRNA expression levels (b) in LPS-stimulated RAW 264.7 cells. (a) Raw 264.7 cells were pretreated with LPS for $2 \mathrm{~h}$, then cells were treated with XF or SBXF water extract in the presence of LPS for another $24 \mathrm{~h}$. NO production was determined by the Griess reagent. (b) Raw 264.7 cells were pretreated with LPS for 2 h, then cells were treated with XF or SBXF water extract in the presence of LPS for another $18 \mathrm{~h}$. iNOS mRNA expression was assessed by real-time PCR. All data were presented as mean $\pm S D$. ${ }^{* *} p<0.01$ vs. control; \&\&p<0.01 vs. LPS; ${ }^{*} p<0.05,{ }^{\# \#} p<0.01$ vs. XF

\section{Statistical analysis}

Each experiment was performed in triplicate and was repeated for at least three times. All results were presented as mean $\pm \mathrm{SD}$. The significance of differences among groups was determined by the one-way analysis of variance (ANOVA). Variance between two groups was evaluated by Student's $t$-test. All analyses were performed using SPSS 17.0 (IBM, USA) with $p<0.05$ as the significance level.

\section{Results and discussion}

Stir-baking reduced the cytotoxicity of XF in MIHA cells It has been reported that XF has liver toxicity in animals [7], and two water-soluble glycosides have been identified to be the toxic substances $[11,12]$. To compare the cytotoxicities of XF and SBXF water extracts, we used the MIHA cell model. Results showed that both XF and SBXF water extracts have no obvious cytotoxicities (data not shown). However, whole power of this herb was also
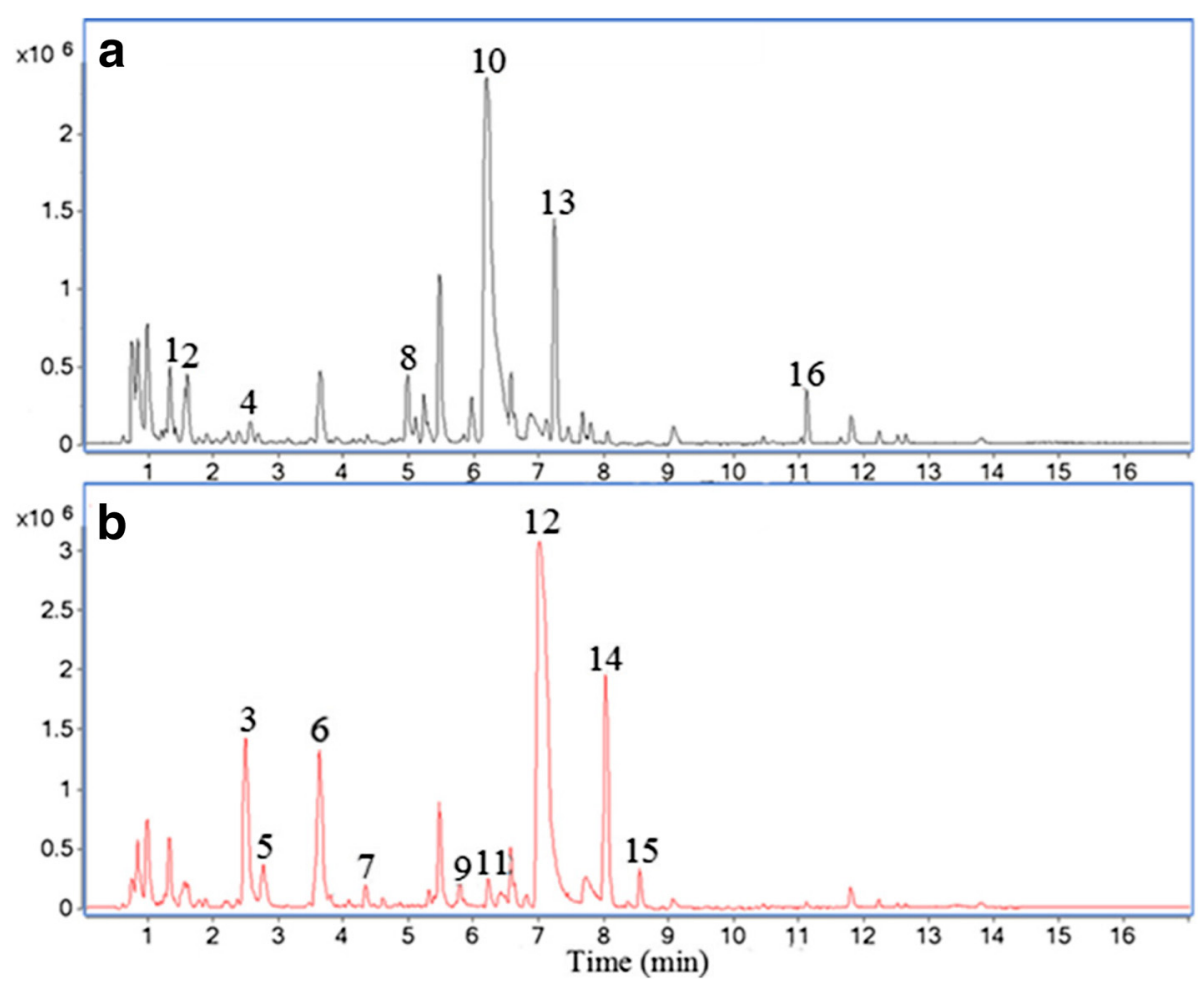

Fig. 3 The representative negative base peak intensity (BPI) chromatograms of XF and SBXF. (a): Chemical profile of XF detected by UPLC/Q-TOF-MS analyses. (b): Chemical profile of SBXF detected by UPLC/Q-TOF-MS analyses 
Table 1 Compounds identified from the water extracts of XF and SBXF

\begin{tabular}{|c|c|c|c|c|c|c|c|c|c|c|}
\hline Peak no. & $t_{R}(\min )$ & Assigned identity & Molecular & $\begin{array}{l}\text { Mean measured } \\
\text { mass (Da) }\end{array}$ & $\begin{array}{l}\text { Mass accuracy } \\
\text { (ppm) }\end{array}$ & $\begin{array}{l}\text { Theoretical exact } \\
\text { mass (Da) }\end{array}$ & $\begin{array}{l}\text { Quasi-molecular } \\
\text { ion }\end{array}$ & $\begin{array}{l}\mathrm{MS} / \mathrm{MS} \mathrm{m} / \mathrm{z} \\
\left(\mathrm{ESI}^{-}\right)\end{array}$ & $\begin{array}{l}\text { Change trend after } \\
\text { processing }\end{array}$ & References \\
\hline 1 & 1.368 & 1-O-caffeoylquinic acid & $\mathrm{C}_{16} \mathrm{H}_{18} \mathrm{O}_{9}$ & 353.0866 & 3.51 & 353.0873 & {$[\mathrm{M}-\mathrm{H}]^{-}$} & 191 & $\downarrow$ & 24 \\
\hline 2 & 1.593 & 3-O-caffeoylquinic acid & $\mathrm{C}_{16} \mathrm{H}_{18} \mathrm{O}_{9}$ & 353.0866 & 8.75 & 353.0873 & {$[\mathrm{M}-\mathrm{H}]^{-}$} & $191 ; 135$ & $\downarrow^{*}$ & 23 \\
\hline $3^{a}$ & 2.487 & chlorogenic acid & $\mathrm{C}_{16} \mathrm{H}_{18} \mathrm{O}_{9}$ & 353.0866 & -1.35 & 353.0873 & {$[\mathrm{M}-\mathrm{H}]^{-}$} & 191 & $\uparrow^{* *}$ & \\
\hline 4 & 2.546 & UN & $\mathrm{C}_{7} \mathrm{H}_{12} \mathrm{O}_{5}$ & 175.0612 & -3.78 & 175.0606 & {$[\mathrm{M}-\mathrm{H}]^{-}$} & & $\downarrow$ & \\
\hline 5 & 2.763 & 4-O-caffeoylquinic acid & $\mathrm{C}_{16} \mathrm{H}_{18} \mathrm{O}_{9}$ & 353.0866 & -6.55 & 353.0873 & {$[\mathrm{M}-\mathrm{H}]^{-}$} & $173 ; 135$ & $\uparrow^{*}$ & 24 \\
\hline $6^{\mathrm{a}}$ & 3.623 & caffeic acid & $\mathrm{C}_{9} \mathrm{H}_{8} \mathrm{O}_{4}$ & 179.0350 & 5.36 & 179.0344 & {$[\mathrm{M}-\mathrm{H}]^{-}$} & & $\uparrow^{* *}$ & 27 \\
\hline 7 & 4.349 & 1,3-O-dicaffeoylquinic acid & $\mathrm{C}_{25} \mathrm{H}_{24} \mathrm{O}_{12}$ & 515.1179 & 3.05 & 515.1190 & {$[\mathrm{M}-\mathrm{H}]^{-}$} & $353 ; 299,173$ & $\uparrow$ & 25,26 \\
\hline 8 & 4.985 & UN & $\mathrm{C}_{25} \mathrm{H}_{24} \mathrm{O}_{13}$ & 531.1144 & -0.36 & 531.1131 & {$[\mathrm{M}-\mathrm{H}]^{-}$} & & $\downarrow$ & \\
\hline 9 & 6.028 & 1,4-O-dicaffeoylquinic acid & $\mathrm{C}_{25} \mathrm{H}_{24} \mathrm{O}_{12}$ & 515.1195 & 8.85 & 515.1190 & {$[\mathrm{M}-\mathrm{H}]^{-}$} & $353 ; 335 ; 179$ & $\uparrow$ & 23 \\
\hline $10^{\mathrm{a}}$ & 6.195 & carboxyatractyloside & $\mathrm{C}_{31} \mathrm{H}_{46} \mathrm{O}_{18} \mathrm{~S}_{2}$ & 769.2110 & 1.23 & 769.2047 & {$[\mathrm{M}-\mathrm{H}]^{-}$} & & $\downarrow^{* *}$ & 12,28 \\
\hline $11^{\mathrm{a}}$ & 6.228 & 1,5-O-dicaffeoylquinic acid & $\mathrm{C}_{25} \mathrm{H}_{24} \mathrm{O}_{12}$ & 515.1299 & -6.07 & 515.1190 & {$[\mathrm{M}-\mathrm{H}]^{-}$} & $353 ; 335 ; 191$ & $\uparrow^{*}$ & 25,26 \\
\hline $12^{\mathrm{a}}$ & 7.03 & atractyloside & $\mathrm{C}_{30} \mathrm{H}_{46} \mathrm{O}_{16} \mathrm{~S}_{2}$ & 725.2155 & -0.62 & 725.2149 & {$[\mathrm{M}-\mathrm{H}]^{-}$} & & $\uparrow^{* *}$ & 28,29 \\
\hline 13 & 7.239 & UN & $\mathrm{C}_{27} \mathrm{H}_{46} \mathrm{O}_{20}$ & 689.2536 & -3.72 & 689.2490 & {$[\mathrm{M}-\mathrm{H}]^{-}$} & & $\downarrow^{* *}$ & \\
\hline 14 & 8.032 & 4'-desulphate-atractyloside & $\mathrm{C}_{30} \mathrm{H}_{46} \mathrm{O}_{13} \mathrm{~S}$ & 645.2594 & -1.05 & 645.2581 & {$[\mathrm{M}-\mathrm{H}]^{-}$} & & $\uparrow^{* *}$ & 29 \\
\hline 15 & 8.575 & UN & $\mathrm{C}_{34} \mathrm{H}_{44} \mathrm{O}_{13}$ & 659.2733 & -3.57 & 659.2691 & {$[\mathrm{M}-\mathrm{H}]^{-}$} & & $\uparrow^{*}$ & \\
\hline 16 & 11.119 & UN & $\mathrm{C}_{19} \mathrm{H}_{30} \mathrm{~N}_{4}$ & 313.2398 & 0.04 & 313.2386 & {$[\mathrm{M}-\mathrm{H}]^{-}$} & & $\downarrow^{* *}$ & \\
\hline
\end{tabular}


used in the clinic, in order to know the cytotoxicities of the components with different polarities, we prepared fractions of an ethanolic extract of XF or SBXF and individually tested their cytotoxicities. As shown in Fig. 1, EA fractions (XF IC $50: 231.1 \mu \mathrm{g} / \mathrm{ml}$; SBXF $\mathrm{IC}_{50}$ : $282.2 \mu \mathrm{g} / \mathrm{ml}$ ) were more toxic than other three fractions (PE fraction: XF $\mathrm{IC}_{50}: 391.8 \mu \mathrm{g} / \mathrm{ml} ; \mathrm{SBXF} \mathrm{IC}_{50}$ : $499.5 \mu \mathrm{g} / \mathrm{ml} ; \mathrm{n}-\mathrm{Bu}$ fraction: XF $\mathrm{IC}_{50}: 271.8 \mu \mathrm{g} / \mathrm{ml}$; SBXF $\mathrm{IC}_{50}: 512.9 \mu \mathrm{g} / \mathrm{ml}$; aqueous fraction: $\mathrm{XF} \mathrm{IC}_{50}$ : $539.9 \mu \mathrm{g} / \mathrm{ml}$; SBXF $\left.\mathrm{IC}_{50}: 560.8 \mu \mathrm{g} / \mathrm{ml}\right)$. Nevertheless, SBXF was less toxic than XF in all four fractions, suggesting that stir-baking reduced the cytotoxicity of XF in MIHA cells.

\section{Stir-baking enhanced the inhibitory effects of XF on NO production in LPS-stimulated macrophages}

$\mathrm{XF}$ is commonly used to treat TCM symptoms that resemble cold, sinusitis and arthritis. It has a good anti-inflammatory activity. In this study, we used LPS-stimulated macrophages as the model to examine if stir-baking alters the anti-inflammatory effects of XF. As shown in Fig. 2, LPS treatment enhanced NO production $(p<0.01) \quad$ (Fig. 2a) and increased mRNA expression of iNOS $(p<0.01)$ (Fig. $2 \mathrm{~b})$ in RAW 264.7 macrophages. Both XF and SBXF water extracts significantly reduced the LPS-elicited NO production $(p<0.01)$ (Fig. 2a) and mRNA expression of iNOS $(p<0.01)$ (Fig. 2b) in a dose-dependent manner. Interestingly, when compared with XF at $300 \mu \mathrm{g} / \mathrm{ml}$, SBXF at the same concentration was more potent in the inhibition of $\mathrm{NO}$ production $(p<0.01)$ (Fig. 2a) and in the reduction of mRNA expression of iNOS $(p<0.05)$ (Fig. 2b) in the macrophages. NO is known to be synthesized from L-arginine by nitric oxide synthase (NOS) and plays a pivotal role as a proinflammatory mediator in various diseases [16-18]. iNOS is highly expressed in LPS-activated macrophages [19] and plays a role in the development and maintenance of inflammation and pain $[20,21]$. Thus, NO production by iNOS may reflect the degree of inflammation and measurements of these two molecules provide options for assessing the effect of drugs in the inflammatory process [22]. These results showed that stir-baking might enhance the anti-inflammatory effects of XF.

\section{Stir-baking altered the chemical profile of XF}

In an attempt to uncover the underlying chemical basis of the reduced cytotoxicity and the enhanced

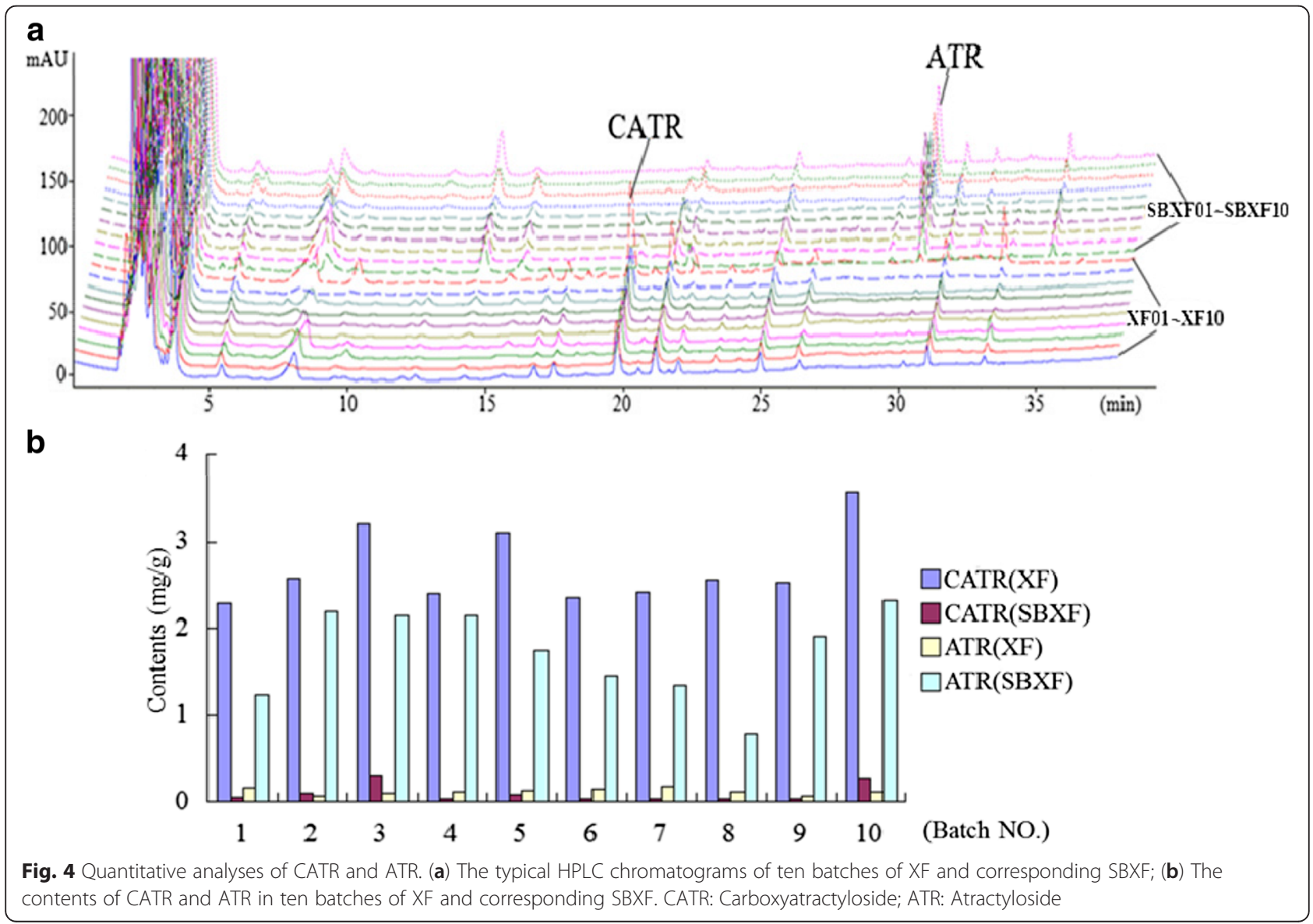


bioactivity caused by stir-baking, we compared the chemical profiles of XF and SBXF by UPLC/Q-TOFMS analyses. As shown in Fig. 3, seven peaks were lower, while nine peaks were higher in SBXF than in XF. Compounds 1, 2, 3, 5 and 7, 9, 11 are two types of isomers, and these compounds were identified by matching with their empirical molecular formulae and mass fragments, as well as the literature data [23-29]. Compounds 3, 6, 10, 11, 12 were confirmed as chlorogenic acid, caffeic acid, carboxyatractyloside, 1,5-O-dicaffeoylquinic acid, and atractyloside by the reference standards. Details of the MS of all components, including phenolic acids and water-soluble glycosides, corresponding to individual peaks were shown in Table 1 . Seven compounds, 1O-caffeotannic acid, 3-O-caffeotannic acid, chlorogenic acid, 4-O-caffeotannic acid, 1,3-O-dicaffeotannic acid, 1,4-O-dicaffeotannic acid and 1,5-O-dicaffeotannic acid have been documented to possess anti-inflammatory effects [23].

It has been reported that two water-soluble glycosides CATR and ATR are the toxic components of this herb $[11,12]$. In this study, we compared the contents of CATR and ATR in ten batches of XF and corresponding SBXF samples using a HPLC method. Results showed that after stir-baking, the content of CATR was decreased 27.0-fold, however, the content of ATR was increased 13.3-fold in this herb. It was reported that CATR is more toxic than ATR in in vitro and in vivo $[30,31]$. Whether CATR in the herb transformed into ATR during stir-baking needs to be further studied. The typical HPLC chromatograms of ten batches of XF and corresponding SBXF were shown in Fig. 4a, and the contents of CATR and ATR in ten batches of XF and SBXF were shown in Fig. 4b.

Chemical and bioactivity studies need to be performed to determine whether the changed compounds are responsible for the reduced cytotoxicity and the enhanced bioactivities of XF after stir-baking.

\section{Conclusion}

We demonstrated that stir-baking significantly reduced the cytotoxicity and enhanced the bioactivity of XF, which support the TCM theory "stir-baking can reduce the toxicity and enhance the efficacy of XF". We have also found that stir-baking caused alterations of components in XF as determined by an established UPLC/Q-TOF-MS method, which might be responsible for the reduced toxicity and enhanced efficacy afforded by processing.

\section{Abbreviations}

ANOVA: analysis of variance; COX-2: cyclooxygenase-2; DMEM: dulbecco's modified eagle medium; ESI: electrospray ionization; iNOS: inducible nitric oxide synthase; NO: nitric oxide; TCM: Traditional Chinese medicine; UPLC/QTOF-MS: ultra-performance liquid chromatography/quadrupole-time-of-flight mass spectrometry.

\section{Competing interests}

The authors declare that they have no competing interests.

\section{Authors' contributions}

The experiments were performed in Dr. YZL's laboratory. Dr. YZL and ST conceived and designed the research; ST and CCY performed experiments; FXQ and LT analyzed data; $\mathrm{GH}$ and $\mathrm{CHH}$ interpreted experimental results; $\mathrm{KHY}, \mathrm{TKW}$ and $\mathrm{YH}$ drafted the manuscript. $\mathrm{CH}$ edited and revised the manuscript. All authors have read and approved the final manuscript.

\section{Acknowledgments}

This work was supported by the Research Grants Council of Hong Kong (HKBU 262512); Food and Health Bureau of Hong Kong (HMRF 11122521); Science, Technology and Innovation Commission of Shenzhen (JCYJ20120829154222473 and JCYJ20140807091945050); and the Hong Kong Baptist University (FRG1/14-15/061 and FRG2/15-16/020).

\section{Author details}

'School of Chinese Medicine, Hong Kong Baptist University, Kowloon Tong, Hong Kong, China. ${ }^{2}$ National Engineering Research Center for Modernization of Traditional Chinese Medicine, Zhuhai, China. ${ }^{3}$ Institute of Integrated Bioinfomedicine \& Translational Science, HKBU Shenzhen Research Institute and Continuing Education, Shenzhen, China.

Received: 12 August 2015 Accepted: 12 January 2016

Published online: 22 January 2016

\section{References}

1. Zhu QS, Zhao WY, Yin QF. Studies on the extraction methods of the antibacterial constituents from Xanthium sibiricum Patr. Qingdao Ke Ji Da Xue Xue Bao. 2008;29:413-8.

2. Chen $B$, Ma LH, Wang XB, Shen YP, Jia XB. Simultaneous determination of 5 phenolic acids in fried Xanthii Fructus from different production sites and its dispensing granules by using ultra-pressure liquid chromatography. Pharmacogn Mag. 2013;9:103-8.

3. Wang RY, Liu XF, Liu MH, Lv WY. The studies of the antioxidant activity and the flavonoids determination of the Xanthii Fructus water extracts. Yunnan Zhong Yi Zhong Yao Za Zhi. 2008;29:42-3.

4. Zhao CS. The study of the antibacterial effects of Xanthii Fructus and its processed products. Shi Zhen Guo Yi Guo Yao. 2002;13:522.

5. Huang MH, Wang BS, Chiu CS, Amagaya S, Hsieh WT, Huang SS, et al. Antioxidant, antinociceptive, and anti-inflammatory activities of Xanthii Fructus extract. J Ethnopharmacol. 2011;135:545-52.

6. Jin CS, Wu DL, Zhang JS. Comparison of the chemical compounds and pharmacological efficacy of different processing methods processed Xanthium sibiricum Patr. Anhui Zhong Yi Yao Da Xue Xue Bao. 2000;19:54-6.

7. Wu B, Cao M, Liu SM. The hepatotoxicity study of the water extract of Xanthium sibiricum Patr. in rats. Yao Wu Bu Liang Fan Ying Za Zhi. 2010;12:381-6.

8. Yan LC, Zhang TT, Zhao JN, Song J, Hua Y, Li L. Comparison of the acute toxicitites of four extracts of Xanthii Fructus in mice. Zhong Guo Zhong Yao Za Zhi. 2012;37:2228-31.

9. Li J, Gao T, Xie ZQ, Tang Y. Comparison of the toxicitites of different extracts of Xanthii Fructus. Shi Zhen Guo Yi Guo Yao. 2005;16:484-7.

10. Gong QF. Science of processing Chinese materia medica. Beijing: Chinese TCM Publishing House; 2013. p. 106.

11. Obatomi DK, Bach PH. Biochemistry and toxicology of the diterpenoid glycoside atractyloside. Food Chem Toxicol. 1998;36:335-46.

12. MacLeod JK, Moeller PD, Franke FP. Two toxic kaurene glycosides from the burrs of Xanthiun pungens. J Nat Prod. 1990;53:451.

13. Levy AS, Simon OR. Six-shogaol inhibits production of tumour necrosis factor alpha, interleukin-1 beta and nitric oxide from lipopolysaccharide-stimulated RAW 264.7 macrophages. West Indian Med J. 2009:58:295-300.

14. Pae HO, Oh GS, Choi BM, Shin S, Chai KY, Oh H, et al. Inhibitory effects of the stem bark of Catalpa ovata G. Don. (Bignoniaceae) on the productions of tumor necrosis factor-alpha and nitric oxide by the lipopolisaccharidestimulated RAW 264.7 macrophages. J Ethnopharmacol. 2003:88:287-91.

15. Chu JH, Wang H, Ye Y, Chan PK, Pan SY, Fong WF, et al. Inhibitory effect of schisandrin B on free fatty acid-induced steatosis in L-02 cells. World J Gastroentero. 2011;17:2379-88. 
16. Colasanti M, Suzuki H. The dual personality of NO. Trends Pharmaco Sci. 2000;13:249-52.

17. Ye H, Xie C, Wu W, Xiang M, Liu Z, Li Y, et al. Millettia pachycarpa exhibits anti-inflammatory activity through the suppression of LPS-induced NO/iNOS expression. Am J Chin Med. 2014;42:949-65.

18. Chae HS, Kang OH, Lee YS, Choi JG, Oh YC, Jang HJ, et al. Inhibition of LPS-induced iNOS, COX-2 and inflammatory mediator expression by paeonol through the MAPKs inactivation in RAW 264.7 cells. Am J Chin Med. 2009;37:181-94.

19. Petros $A$, Bennett $D$, Vallance P. Effect of nitric oxide synthase inhibitors on hypotension in patients with septic shock. Lancet. 1991;338:1557-8.

20. Jeong JW, Lee HH, Han MH, Kim GY, Hong SH, Park C, et al. Ethanol extract of Poria cocos reduces the production of inflammatory mediators by suppressing the NF-kappa B signaling pathway in lipopolysaccharidestimulated RAW 264.7 macrophages. BMC Complement Altern Med. 2014;15:101.

21. Levy D, Hoke A, Zochodne DW. Local expression of inducible nitric oxide synthase in an animal model of neuropathic pain. Neurosci Lett. 1999;260:207-9.

22. Naureckien S, Edris SK, Ajit SK, Katz AH, Sreekumar K, Rogers KE, et al. Use of a murine cell line for identification of human nitric oxide synthase inhibitors. J Pharmacol Toxicol Methods. 2007:55:303-13.

23. Han T, Li HL, Zhang QY, Han P, Zheng HC, Rahman K, et al. Bioactivityguided fractionation for anti-inflammatory and analgesic properties and constituents of Xanthium strumarium L. Phytomedicine. 2007;14:825-9.

24. Morishita H, Iwahashi H, Osaka N, Kido R. Chromatographic separation and identification of naturally occurring chlorogenic acids by ${ }^{1} \mathrm{H}$ nuclear magnetic resonance spectroscopy and mass spectrometry. J Chromatogr. 1984;315:253-60

25. Clifford MN, Knight S, Kuhnert N. Discriminating between the six isomers of dicaffeoylquinic acid by LC-MS ${ }^{n}$. J Agric Food Chem. 2005;53:3821-32.

26. Clifford MN, Wu W, Kirkpatrick J, Kuhnert N. Profiling the chlorogenic acids and other caffeic acid derivatives of herbal chrysanthemum by LC-MS ${ }^{\text {. }}$. J Agric Food Chem. 2007;55:929-36.

27. Sandra P, Pasquale C, llaria B, Donato A, Aldo DL, Marco E, et al. HPLC-DADMS/MS characterization of phenolic compounds in white wine stored without added sulfite. Food Res Int. 2014:66:207-15.

28. Carlier J, Romeuf L, Guitton J, Priez-Barallon C, Bévalot F, Fanton L, et al. A validated method for quantifying atractyloside and carboxyatractyloside in blood by HPLC-HRMS/MS, a non-fatal case of intoxication with Atractylis gummifera L. J Anal Toxicol. 2014;38:619-27.

29. Sonia P, Cosimo P, Nunziatina DT, Francesco DS. Sesquiterpene and diterpene glycosides from Xanthium pungens. Phytochemistry. 1996;41: 1357-60.

30. An J, Wang YD, Sheng CC. Comparative analysis of the contents of carboxyatractyloside and atractyloside in Xanthii Fructus and its processed products. Yao Wu Fen Xi Za Zhi. 2013;33:1910-3.

31. Yu J, Song MZ, Wang J, Li YF, Lin P, Que L, et al. In vitro cytotoxicity and in vivo acute and chronic toxicity of Xanthii Fructus and its processed product. Biomed Res Int. 2013:403491-503.

\section{Submit your next manuscript to BioMed Central and we will help you at every step:}

- We accept pre-submission inquiries

- Our selector tool helps you to find the most relevant journal

- We provide round the clock customer support

- Convenient online submission

- Thorough peer review

- Inclusion in PubMed and all major indexing services

- Maximum visibility for your research

Submit your manuscript at www.biomedcentral.com/submit 